\title{
DEFENSINS AND CYTOKINS IN NAIL UNIT AFFECTED BY ONYCHOMYCOSIS: A PILOT STUDY
}

\author{
Olga Zaikovska ${ }^{1}$, Janis Kisis ${ }^{1}$, Mara Pilmane ${ }^{2}$ \\ ${ }^{1}$ Department of Infectology and Dermatology, Riga Stradinš University, Latvia \\ ${ }^{2}$ Institute of Anatomy and Anthropology, Riga Stradinš University, Latvia
}

\begin{abstract}
Onychomycosis is a chronic infection disease that is induced by different types of fungi. Changes of nail color, shape and thickness are disease clinical findings. The disease affects all ages and genders of people, however higher incidence is observed in the people who have reduced immunity.

To study natural immunity of the nail, 22 nail biopsies from onychomycosis patients were taken. Material was stained with hematoxilin and eosin and immunohistochemical methods were used.

We found the nests of hypertrophy in the granulose layer of the epidermis, with focal acanthosis. In the horn layer, we identified the nests of parakeratosis of various sizes, with the incorporations of homogenous and eosinophil masses.

We found high levels of IL- 6 and IL-10 positive cells in the bed of the nail and in the bloodstream. IL-1, however, was not a part of any of the functional units of any of the nails. High amount hBD-2-containing fibres was found in the bed and the plate of the nail. Interestingly, hBD-3 and hBD-4 were not found in any of the samples.

So, when it comes to onychomycosis infected nails, the most effective morphopathogenic processes include cytokine and defensin excretion occurs in the bed of the nail.
\end{abstract}

Keywords: onychomycosis, nail biopsy, antimicrobial peptides, defensins, interleikins 


\section{INTRODUCTION}

Onychomycosis is one of the most common nail diseases. It is a chronic nail infection induced by different types of fungi: dermatophytes, moulds and yeast. The clinical picture is characterized by changes in the nail plate colour, shape and thickness.

The disease affects people of all ages and genders, however higher incidence is observed in the people with reduced immunity. Usually they are elderly, diabetic, eczematic or psoriasis patients, as well as patients undergoing immunosuppression $[6,19,3,2]$.

Recently researchers have actively studied the natural antimicrobial defence system of the organism. There are many studies describing the role of natural immunity in infection fighting process. Even though skin is exposed to microorganisms daily, no contamination or infection develops due to the epithelial defence system. The skin protects itself from infection by various mechanisms. The antimicrobial peptides produced by keratinocytes combined with unique skin ions, lipids and physical barrier, perform a very important role in skin protection.

There are many antimicrobial peptides (AMPs), some discovered particularly in the human skin. These peptides have antibacterial, antiviral and antifungal properties. It is a unique group of molecules, which are divided into several subgroups, depending on the amino acid structure. AMP is a small molecule, which has both hydrophilic and hydrophobic nature. AMP may inhibit or kill microbes selectively damaging their cell membranes. As the most important antimicrobial peptides in humans, cathelicidin and the family of defensins are mentioned $[11,15,9]$.

Cathelicidins consist of 12 to 80 amino acids. The human cationic antimicrobial peptide (hCAP18) is the only one identified human cathelicidin to date. Besides neutrophils the hCAP18 was also found on monocytes, lymphocytes, squamous epithelium cells (mouth, tongue, oesophagus, cervix and vagina), lung epithelium and in keratinocytes. The antibacterial activity of C terminal fragment of hCAP18 - LL37 (37 aminoacids) against Gr + and Gr- bacteria, fungi, viruses, protozoa was established. This peptide shows the synergistic antibacterial effect with defensins. LL-37 attracts lipopolysaccharide and neutralizes its ability to induce endotoxic shock. This peptide is an important factor in the re-epitalisation of the wound. The angiogenic activity of LL-37 was 
demonstrated both, in vivo and in vitro. In addition, LL-37 acts as the factor of hemotaxis for neutrophils, monocytes and T cells [13] .

Defensins are small, cationic peptides acting on the micro-organisms by damaging the membrane permeability via forming ion channels. There are 2 main groups of mammalian defensins: alpha and beta defensins. Beta defensins are synthesized only by neutrophils, thus considered the line of specific markers of these cells. The activation of neutrophils to inflammation and infection leads to the rapid release of defensins, which are then found in plasma and other body fluids. In normal plasma very low numbers of defensin are found, but in the conditions of sepsis, the numbers of defensin rise. In addition to the defensin antimicrobial properties, AMPs are showing activity in hemotaxis, immunomodulation and cytotoxic processes, that is essential to the common body defense and inflammation development. There is evidence that the patients who have lack of defensins, suffer from frequent and severe bacterial infections [17].

In general, humans have 9 epithelial defensin types, 4 of which are beta defensins [1-4]. Beta defensin 1 (hBD-1) is expressed constantly, but hBD-2, hBD-3 and hBD-4 expression induced by peptides. This is considered as an exclusive component of the mucosal anti-microbial innate immunity component $[7,18]$.

These peptides work as efectormolecules, as well as the immune response, inflammatory response and tissue healing processes regulators $[13,14]$.

There have been numerous studies on the presence of antimicrobial peptides and immune processes in the skin and mucosa. However, there is very limited information available specifically on the ongoing processes in the nail. In this study we have detected the human beta-defensins and inflammatory cytokines in onychomycosis patient nails.

\section{MATERIAL AND METHODS}

\section{Material}

In order to evaluate morphological and immunohistochemical picture of onychomycosis, nail $5 \mathrm{~mm}$ punch biopsies from nail were taken [8] . The study included 22 patients corresponding to the pre-established selection criteria to the exclusion of a variety of possible factors influencing the results (a previous drug therapy, the nail condition affecting diseases). Eligible patients were aged 
from 31 to 81 years with clinically and microscopic/culture confirmed diagnosis of onychomycosis. Onychomycosis clinical severity was determined by the onychomycosis clinical severity index method (the Scoring Clinical Index for Onychomycosis). The SCIO value ranged from 12 to 30 , corresponding to severe onychomycosis.

The study was approved by the Ethical Committee at Riga Stradins University, the permit issued on 26.01.2012.

\section{Method}

1) $5 \mathrm{~mm}$ punch nail biopsies were fixed in Stefanini's solution, dehydrated and embedded in paraffin. Four micrometer-thick sections were prepared from each tissue specimen and stained routinely with hematoxylin and eosin.

2) The immunohistochemical method. Tissue was determined for the human beta defensin-2 (VJU01, 1:100, RD), human beta defensin-3 (NB200-117, 1:1000, USA), human betadefensin-4 (L1310-D1, 1:100, California, USA), human 1alfa interleukin (B-7SC-9983, 1:50, California, USA), interleukin 6 (NYRhIl-6, 1:50, California, USA) and interleukin 10 (AB34843, 1:400, England).

3) For the visual illustration of our findings we used Leica DC 300F digital camera and image processing and analysis software Image Pro Plus. The intensity of immunostaining was graded semi-quantitatively. Few positive structures in the visual field were labelled with + , the moderate number of positive structures in the visual field was labeled with ++ , numerous positive structures in the visual field were labeled with +++ , and the abundance of positive structures in the visual field was marked with ++++ (M. Pilmane, 1998).

\section{RESULTS}

We found patchy hypertrophy in the granulose layer of the epidermis and focal acanthosis in all the cases (Figure 1). In stratum corneum, parakeratosis and incorporated eosinophil homogenous masses were observed (Figure 2).

Numerous hBD-2 containing fibres were found in the bed of the nail (Figure 3 ), but the moderate number of $\mathrm{hBD}-2$ positive cells was found in the underlying connective tissue and the nail plate. hBD-3 and hBD-4 were not found in preparations. 


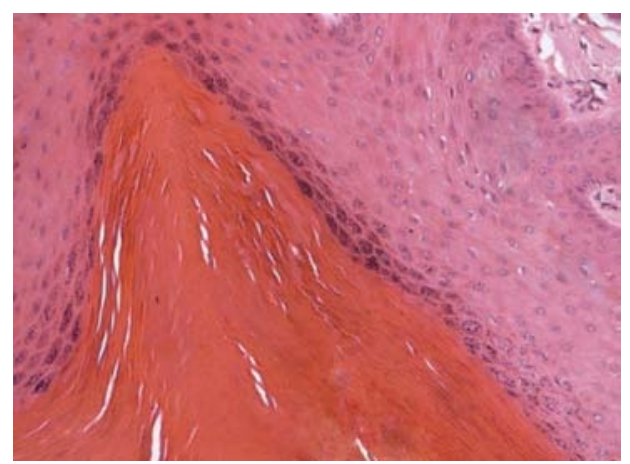

Figure 1. Hypertrophy of the epidermis, with focal acanthosis. Hematoxyllin and eosin, X200

Hematoxyllin and eosin, X200.

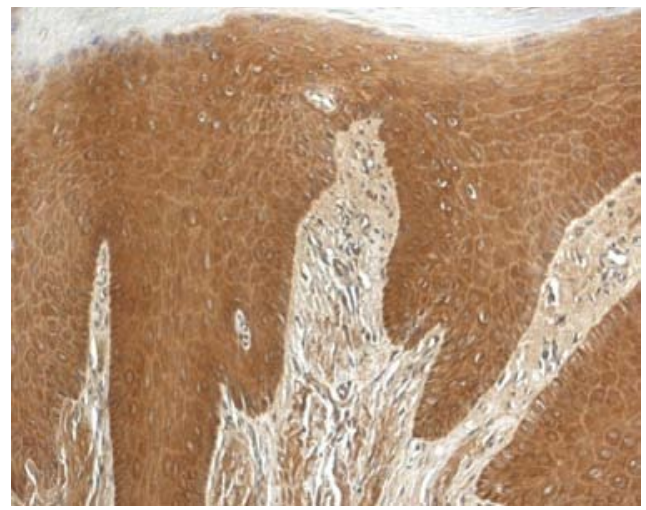

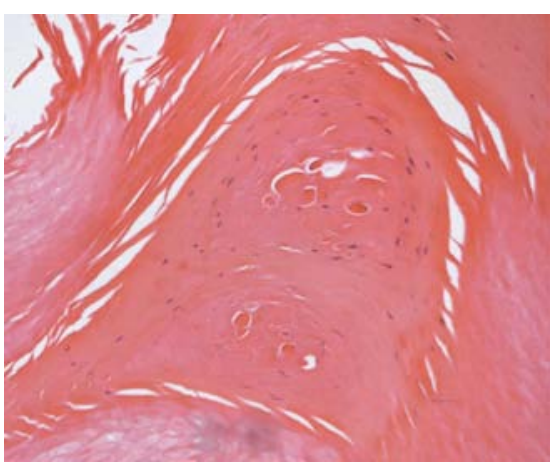

Figure 2. Parakeratosis with incorporated homogenous and eosinophil masses in stratum corneum.

Figure 3. High expression of HBD-

2 in the nail bed and the underlying connective tissue. hBD-2, IMH, X200.

Few IL-1 positive structures were detected in 6 preparations, in particular in the nail bed. Numerous IL- 6 positive cells were found in the nail bed and smooth muscles of blood vessels (Figures 4-5), but just few positive cells were found in the plate structure. Moderate IL-10 containing structures were found in the nail bed, but in the connective tissue and the plate structure there were few positive cells (Figures 6-7).

The overview of all the staining semi-quantitative results is available in Table 1. 


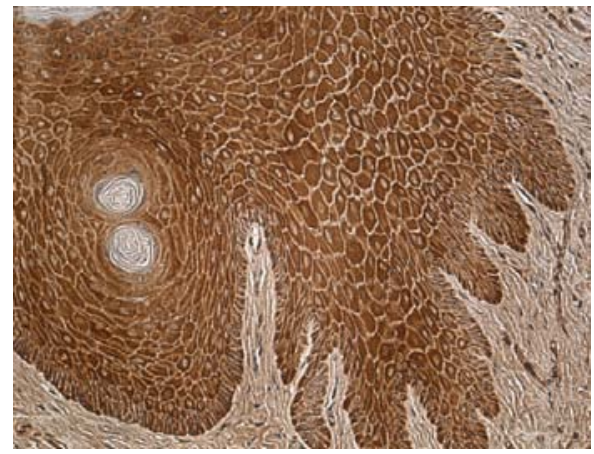

Figure 4. Abundance of IL-6 positive keratinocytes in the nail bed. IL-6, IMH, X200.

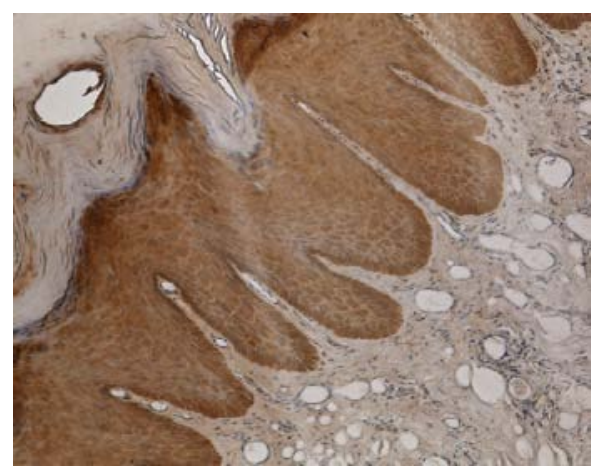

Figure 6. Numerous IL-10 positive keratinocytes in the nail bed. IL-10, IMH, $\mathrm{X} 100$.

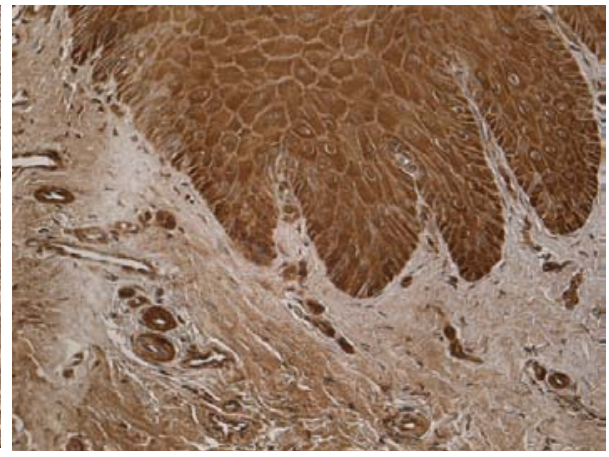

Figure 5. Abundance of IL-6 containing endothelial cells and smooth muscle in blood vessels in the nail bed. IL-6, IMH, X 200.

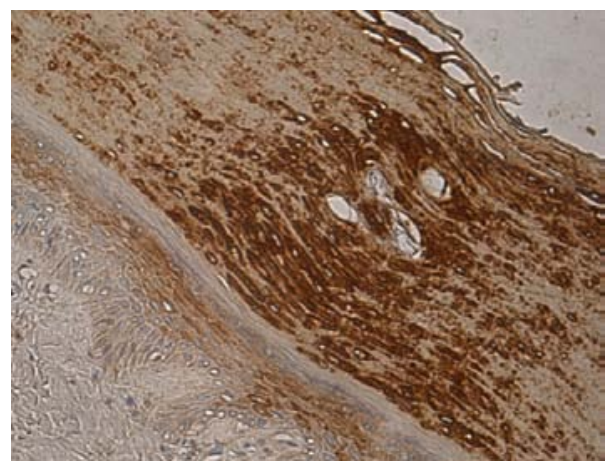

Figure 7. Moderate number of IL-10 positive cells in the nail plate structure and few positive cells in the nail bed. IL-10, IMH, X 200.

Table 1. Overview of all staining sem-quantitative results.

\begin{tabular}{cccc}
\hline Staining & Nail bed & Connective tissue & Nail plate \\
\hline hBD-2 & $++/+++$ & ++ & $+/++$ \\
\hline hBD-3 & 0 & 0 & 0 \\
\hline hBD-4 & 0 & 0 & 0 \\
\hline IL-1 & $0 /+$ & $0 /+$ & $0 /+$ \\
\hline IL-6 & +++ & $+/++$ & + \\
\hline IL-10 & $+/++$ & + & +
\end{tabular}

Designation:

0 negative reaction

0/+ positive few elements in some preparations

$+\quad$ few positive structures in the visual field

++ moderate number of positive structures in the visual field

+++ numerous positive structures in the visual field

++++ abundance of positive structures in the visual field 


\section{DISCUSSION}

If microbes overcome the epidermal skin barrier, then on the next line of defense there are macrophages, neutrophils and inflammatory reaction in the tissue. On the surface of macrophages, epithelial and dendritic cells, there are transmembrane receptors (PRRS Pattern Recognition-receptor) that recognize the different types of the molecular determinants that are associated with pathogenic and non-pathogenic bacteria. The most prominent of these receptors are Toll-like (TLRs) and mannose receptors. Depending on which receptor is linked to the determinant, a variety of reactions are expected. The receptor recognizes the nature of the pathogen and releases the response mechanism, mostly with the help of cytokines. The cytokines involved in the response reaction via TLR are tumor necrosis factor alfa, IL-1, IL-6 [1, 20, 4] .

During the present study, IL-1 was not found in any of the nail unit structures in all the preparations in sufficient quantities. However, significant quantities IL-6 were found in the nail bed structures in particular. IL- 6 is an interleukin that acts as both a pro-inflammatory and an anti-inflammatory cytokine. It is secreted by $\mathrm{T}$ cells and macrophages to stimulate immune response, during and after injury, especially in the burns and other tissue damages leading to inflammation. The role of IL- 6 as an anti-inflammatory cytokine is mediated through its inhibitory effect on TNF-alpha and IL-1, and the activation of the IL-1ra and IL-10.

It is significant that the IL-10 was found in large quantities in the studied preparations. IL-10 is an anti-inflammatory cytokine, produced primarily by monocytes and to a lesser extent by lymphocytes. This cytokine has a pleiotropic effect in immunoregulation and inflammation. It down-regulates the expression of Th1 cytokines, MHC class II antigens and costimulatory molecules on macrophages. It also enhances B cell survival, proliferation, and antibody production [12].

hBD-2 was found in very large quantities in this work exploring the presence of natural antimicrobial peptides in the nails affected by onychomycosis. However, hBD-3 and hBD-4 were not found in significant quantities. hBD-2 plays an important role in hemotaxis, also it induces the maturation of dendritic cells in a TLR4 dependent manner. Moreover, hBD-2 has shown the ability to induce intracellular $\mathrm{Ca}^{2+}$ mobilization and histamine release in mast cells, probably contributing to the inflammatory and vasodilatory responses enhancing the induction phase of adaptive immunity [14] . 
According to previous findings $[16,12]$, our data are controversial with the other authors' data that show hBD-2 expression in the infected skin inducted by IL-1.

It is important that there are studies on nails and studies related to pathogenic fungi infections also available. Dorschner and co-authors studied the nail unit of the natural innate immunity point of view, showing mouth extract antimicrobial activity against Candida albicans [5].

Our study showed similar histological findings with pathogenic fungi induced changes in the skin. A similar study was carried out with the mycotic infection of the smooth skin. Histological sections showed the involvement of all epidermal layers in the reaction against dermatophytes in the form of epidermal thickening due to hyperkeratosis and akanthosis. In the upper dermis the lymphocytes and neutrophils infiltration was detected. In this study, the presence of the human hBD-2 in the skin affected by dermatophytes, as well as the importance of this AMP activity against Trichophyton rubrum was established [10] .

Keratinocytes, in particular, were identified as the main cellular sources of antimicrobial peptides produced during inflammatory processes in the skin. Expression levels of these natural antibiotics correlate well with susceptibility to skin infections. Thus, the correlation between the AMP expression and commensal microbiota may be very important to maintaining skin homeostasis.

\section{CONCLUSIONS}

1. IL-6 and IL-10 are most common cytokins in the nails affected by onychomycosis.

2. All the structures expresses the antimicrobial peptide defensin -2 in moderate numbers in the nail unit affected by onychomycosis.

\section{REFERENCES}

1. Bahri R. (2010). Candida famata modulates toll-like receptor, beta-defensin, and proinflammatory cytokine expression by normal human epithelial cells. J Cell Physiol, 222, 1, 209-18.

2. Bouguerra R. (2004). Prevalence and clinical aspects of superficial mycosis in hospitalized diabetic patients in Tunisia. Med Mal Infect, 34, 5, 201-5.

3. Cordeiro R. A. (2006). Antifungal susceptibility and genetic similarity of sequential isolates of Trichophyton rubrum from an immunocompetent patient with chronic dermatophytosis. Clin Exp Dermatol, 31, 1, 122-4. 
4. De Koning H. D.( 2012). Pattern recognition receptors in infectious skin diseases. Microbes Infect, 14, 11, 881-93.

5. Dorschner R. A. (2004). Innate immune defense of the nail unit by antimicrobial peptides. J Am Acad Dermatol, 50, 3, 343-34.

6. Faergemann J. (2005). Genetic predisposition-understanding underlying mechanisms of onychomycosis. J Eur Acad Dermatol Venereol, 19, 1, 17-9.

7. García J. R. (2001). Human beta-defensin 4: a novel inducible peptide with a specific salt-sensitive spectrum of antimicrobial activity. FASEB J, 15, 10, 1819-21.

8. Grover C. (2005). Nail biopsy: assessment of indications and outcome. Dermatol Surg, 31, 2, 190-194.

9. Izadpanah A. (2005). Antimicrobial peptides. 52, 1, 381-90.

10. Jensen J. (2007). Barrier function, epidermal differentiation, and human betadefensin 2 expression in tinea corporis. J Invest Dermatol, 127, 7, 1720-1727.

11. Lai J. (2010). AMPed Up immunity: how antimicrobial peptides have multiple roles in immune defense. Clin Immunol, 135, 1, 1-11.

12. Liu A. Y. (2002). Human beta-defensin-2 production in keratinocytes is regulated by interleukin-1, bacteria, and the state of differentiation. J Invest Dermatol, 118, 2, 275-81.

13. Nakatsuji T. (2012). Antimicrobial peptides: old molecules with new ideas. J Invest Dermatol, 132, 2, 887-95.

14. Pivarcsi A. (2005). Innate immunity in the skin: how keratinocytes fight against pathogen. Current immunology reviews, 2005, 1, 29-42.

15. Rodrigues E. G. (2009). Antifungal and antitumor models of bioactive protective peptides. An Acad Bras Cienc, 81, 3, 503-20.

16. Schauber J. (2009). Antimicrobial peptides and the skin immune defense system. J Allergy Clin Immunol, 124, 2, 13-8.

17. Schröder J. M. (2010). The role of keratinocytes in defense against infection. Curr Opin Infect Dis, 23, 2, 106-110.

18. Smiley A. K. (2007). Expression of human beta defensin 4 in genetically modified keratinocytes enhances antimicrobial activity, 28, 1, 127-32.

19. Szepietowski J. C. (2007). Do fungi play a role in psoriatic nails? Mycoses, 50, 6, 437-42.

20. Sørensen O. E. (2005). Differential regulation of beta-defensin expression in human skin by microbial stimuli. J Immunol, 174, 8, 4870-9.

\section{Address for correspondence:}

Olga Zaikovska

Department of Infectology and Dermatology

Riga Stradiņš University

Rìga, Kāḷu 25, LV1058

E-mail: dr.zaikovska@gmail.com 